\title{
FROM A TESTING LABORATORY FOR RAILWAY TECHNICAL COMPONENTS TO A HUMAN FACTORS SIMULATION ENVIRONMENT: $3^{\text {RD }}$ INTERNATIONAL RAIL HUMAN FACTORS CONFERENCE
}

\author{
Malte Hammerl, Monica De Filippis, Ingo Steinhäuser, Christoph Torens, \\ Oliver Gantz, Michael Meyer zu Hörste, Karsten Lemmer
}

\author{
German Aerospace Center \\ Institute of Transportation Systems \\ Lilienthalplatz 7 \\ 38108 Braunschweig, Germany \\ malte.hammerl@dlr.de \\ monica.filippis@dlr.de \\ ingo.steinhaeuser@dlr.de \\ christoph.torens@dlr.de \\ oliver.gantz@dlr.de \\ michael.meyerzuhoerste@dlr.de \\ karsten.lemmer@dlr.de \\ Phone: + 495312953401 \\ Fax: $\quad+495312953402$
}

\begin{abstract}
Due to continuous automation and the introduction of new technologies at railway workplaces, there is a high demand for human factors research in the railway domain. In order to study the human contribution to performance and safety of the railway system, the Institute of Transportation in the German Aerospace Centre decided to upgrade the existing railway laboratory RailSiTe to a human factors simulation environment.

As a result, valid subject studies for train drivers can be run without risk for life and material and do not interfere with real railway operations. This paper describes, how the original simulator - designed to test technical components for the European Train Control System - was reconfigured to meet the requirements for human factor studies. The chosen criteria are described as well as the possibilities of the new environment.
\end{abstract}

Keywords: Human factors, Railway, Simulation, Laboratory, Train driver

\section{Introduction}

The approach of integrating human factors is still not common practice in railway engineering in continental Europe. This is critical as the development in the last years have transformed railway workplaces to less executing but more supervisory environments. The physical work has been replaced by cognitive tasks, typical of a modern human-machine-interaction. Years of continuous automation have targeted at the reduction or a control of human contribution. But unfortunately, accidents persist and are to a high percentage accounted to human error.

Here, human factors research in the railway domain strives for a better humanmachine-interaction for a well-performing and safe railway system. Instead of automation, user-centred-design represents a suitable approach for human factors integration. Particularly, in Germany, less literature on railway human factors can be found. But, information on how train driver behave, perform and interact with the system in certain conditions is valuable for future improvements of the humanmachine-interface (HMI).

The need for further research motivated the Institute of Transportation in the German Aerospace Center to emphasize the analysis of rail human factors. The existing RailSiTe railway laboratory has been upgraded to a human factors simulation environment. The next sections of the paper describe the reasons and the 
scope of the improvements.

\section{Advantages and drawbacks of a simulation environment}

In general, simulations offer the possibility to conduct research without risk for life and material. Additionally, studies do not interfere with real railway operations. It is obvious that the maintenance of the simulation environment is less expensive than a similar arrangement in practice. In our case, the simulator RailSiTe is furthermore dedicated for research and not dependent on a railway undertaking. Training programmes do not impair the availability of the simulation environment.

As all simulations, the RailSiTe permits to build characteristics on-top that could not be studied in real-world. Fictitious railway networks can be constructed. With this feature, monotony, workload, fatigue and stress can be induced as relevant for the study. With special configuration, also sudden events, for example at a level crossing, can be introduced and studied easily.

Finally, the simulator allows for the control of interfering variables and manifold studies: evaluation of re-design of different interfaces, the testing of new driving assistant systems, comparison of signalling systems etc.

All these advantages are opposed by the possible criticism that any result is not based on a real-world study but the simulation in an artificial environment. There is always the risk to neglect a possibly important detail. That is why simulations are proposed as a primary analysis before tests in real world. But in any case, human factors studies in simulation environments show valid results in other industry domains and are as such also valuable for railway transport.

\section{Laboratory architecture}

The DLR's railway specific laboratory RailSiTe had been designed from the beginning to fulfill a large variety of different requirements. One important purpose had been the capability to test components of train control systems regarding conformity to their specifications and interoperability with components of other arbitrary suppliers. The introduction of the common European train control system ERTMS/ETCS had been one of the main motivations for that (Jaschke et al. 2007).

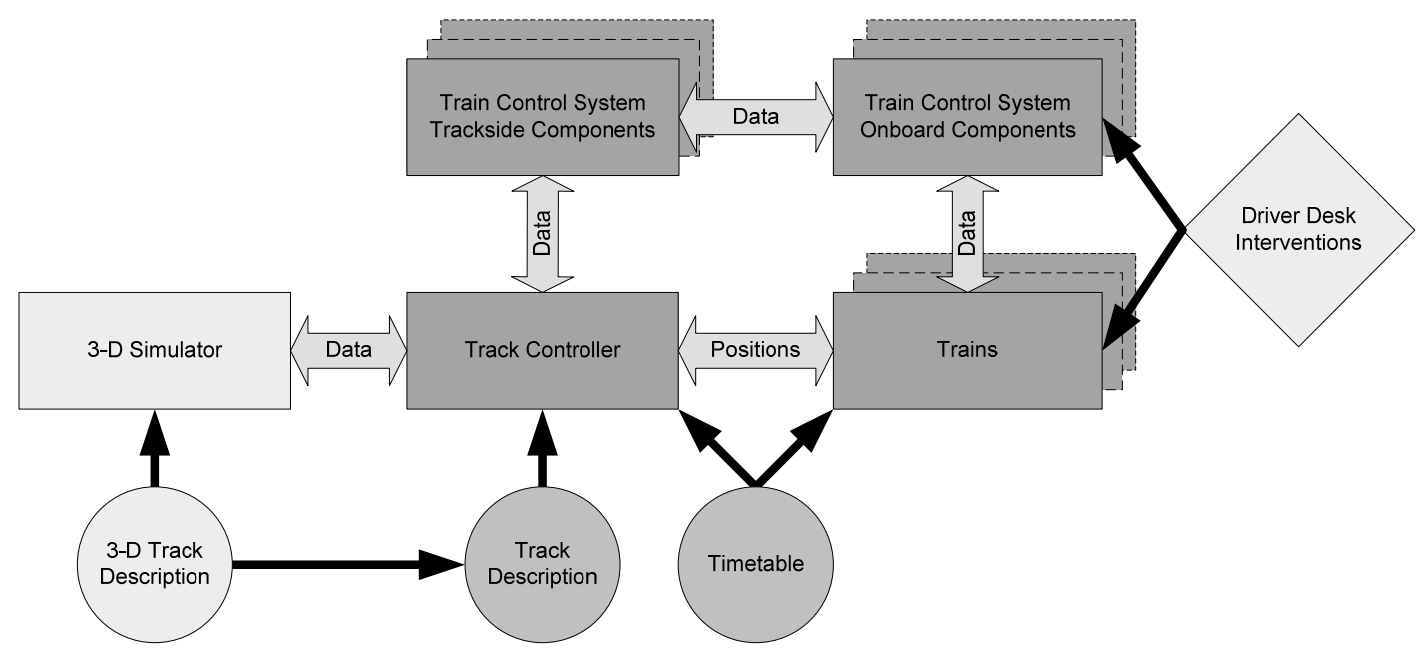

Figure 1: Laboratory architecture with updates for human factors studies

The track description data containing e.g. tracks, switches, signals, block sections and trackside transponder positions are stored in a central database. On simulation start, these data are transmitted to the track controller, a central simulation component which frequently collects and dispatches all dynamic simulation parameters, such as train positions, train dynamics, switch positions and signal aspects. An arbitrary number of trains can be moved independently on the 
given track layout by predefined timetable and speed profile information stored in a database. The train modules simulate the physical movement of the masses according to their acceleration and braking characteristics. The train simulations are connected to their corresponding train control system onboard unit, which may be simulated or an industrial component, and is interchanging data with the wayside equipment.

So, the RailSiTe is fully capable of simulate realistic train routes and movements in a railway network. A diagram of the modular architecture is shown in figure 1; the core laboratory equipment is show in the center, in a darker shade. The figure already illustrates some of the major software modifications for the extension for human factors studies. A driver's desk and a visualisation of the train driver's view were available at the beginning of the update for human factors studies (see extensions in light shade in figure 1). Driver desk interventions represent the manual control of one of the trains. So, physically, a driver's cabin had to be constructed and a supervisor desk had to be created. In order to establish a realistic train driver's environment, the driver-machine-interface and the sound system had to be updated. Finally, the data logging had to be modified to be able to record video data as well as physiological and eye-movement measurements. The updates are described in the next section.

The institute disposes of a movement platform that is currently equipped with a car simulator. Due to the predominantly monodirectional accelerations in the railway applications and due to cost reasons, the installation of the railway driver's cabin on the movement platform was decided to be part of a future step. Additionally, the implementation of the German continuous train control system "LZB" was postponed. The availability of the conventional intermittent automatic train control "PZB" was regarded as primarily vital. Note that the European system ETCS was natively incorporated in the RailSiTe laboratory environment.

\section{Description of modifications in detail}

This section illustrates the updates of the laboratory. Thereby, the requirements for human factors studies, the necessary modifications and the final capabilities of the implemented solution are addressed.

\subsection{Track preparation and visualisation}

Within the RailSiTe, the commercial train simulator ZUSI is responsible for the visualisation. A principal implementation was already available before the updates for human factors studies.

However, the degree of realism of the scenery shown to the driver is directly related to the test results. Therefore, a high level of realism is needed - a lot of detail design work for the visualisation of the railway track and surrounding landscape has to be foreseen. This goal can be reached by modelling a real or a fictitious railway line. To perform tests with an estimated duration of half an hour, a total track length of at least 50 kilometres is required.

The track data and visualisation are kept in two separate data formats. The first format is the track description related to the RailSiTe modules, needed for the realistic control of train route setting and vehicle movements. The ZUSI track visualisation represents a second database. Naturally, certain track data in the two formats has to be consistent to enable data exchange. For example, the visualisation has to change a signal aspect on demand when the route has been set in the RailSiTe control centre.

Unfortunately, the creation of 3D visualisation in ZUSI format from scratch involves a complex effort. That is why a $50 \mathrm{~km}$ track including all landscape objects was purchased from the supplier. All landscape design, such as vehicles, houses, surfaces and other objects are at free disposal for the future creation of new railway lines. 
For the edition of lines for the RailSiTe format, the institute disposes of a track editor. Subsequently, the railway line configuration can be imported to ZUSI, the landscape visualisation data will be added with a separate visualisation editor. In order to facilitate the track construction process, import and export tools have been developed. For example, the laboratory disposes of the possibility to import track configurations from the international format RailML (Knollmann et al., 2005; Nash et al., 2004)

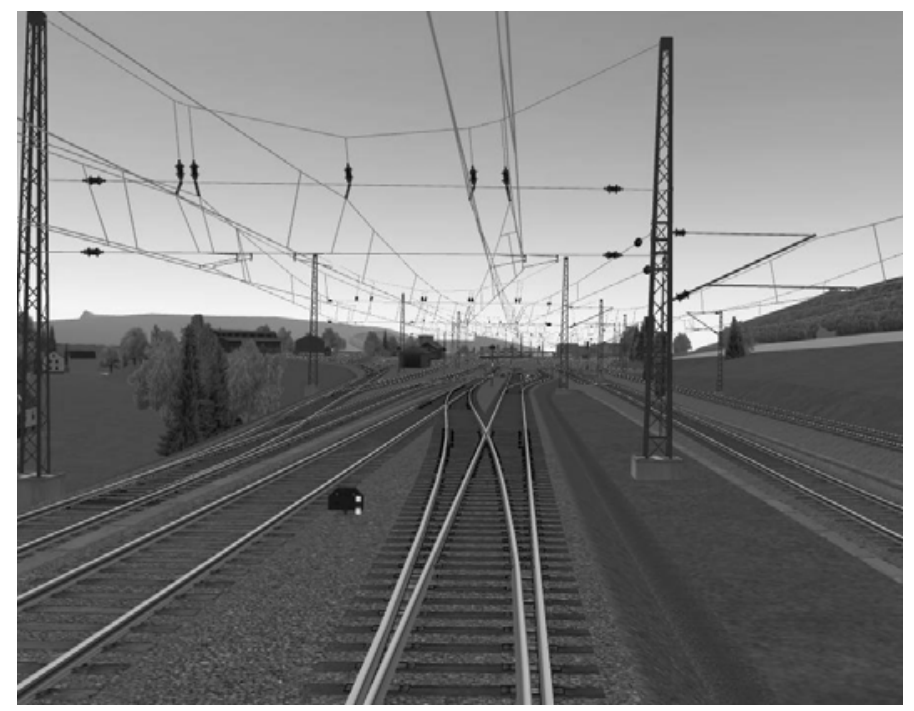

Figure 2: Example for a visualisation

\subsection{Sound environment}

For an immersive driving experience during the subject studies, a sound environment had to be created. All sounds that have their origin in direct actions of the driver had to be implemented, e.g. accelerating, braking, the horn, raising or lowering the pantograph. Additional sounds particular for the locomotive series should be added in a later version, e.g. the running steps, relays or ventilators.

Fortunately, the visualisation set incorporates native support for 3D sound environments. In order to not only have speed-dependent sound effects but also simulate driver-activated acoustical events, an interface between the laboratory's driver desk and Zusı had to be implemented. Enabling the data transfer with low latencies required enhancements in both components ZuSI and the driver's desk interface. The sound profiles for the different traction units were purchased from third-party who works closely with train drivers to maximize the level of realism. The sound system was installed in the driver's cab described later in this paper.

\subsection{Supervisor desk and data logging}

Section 3 showed that the RailSiTe laboratory is a highly distributed computer network with specialized modules for every aspect of the simulated environment of a full functional rail system. To be used in human factors studies, first, the handling of the simulator had to be usable for a single psychological supervisor and independent from highly specialized technical staff. Second, different studies required additional information about the actions and performance of the subject to be made available at the supervisor desk: communication with the user had to be facilitated and recorded; also video and audio signals had to be logged. Additionally, an on-line monitoring of the track and the actions of the driver as well as the possibility to manipulate the track signalling had to be made possible.

One of the major critical aspects of a simulator usable for human factors studies is the sensible logging of the obtained data. Also, the problem of synchronisation of 
heterogeneous data types had to be solved to guarantee meaningful data analysis.

To meet all the listed requirements, the supervisor desk is designed to be an allaware surveillance monitor (figure 1). The integration of the most relevant RailSiTe software modules to a central desktop station was made possible. The most important modules are the start-up module, the interlocking simulator and the auxiliary train control module. These software modules run on a Linux-PC that the supervisor desk is primary based on. The supervisor has a view of the electronic time table (EBuLa), the driver-machine interface (DMI) and the driver-desk-interface (DDI) that encompasses all input buttons and levers; all controlled by the RailSiTe.

Furthermore, a Windows-PC runs the psychological software application E-Prime and the audio capture software. The videos are captured by a standalone digital video recorder. Finally, the large observation display on top serves the surveillance of the subject and the duplication of the 3D-visualisation the driver sees.

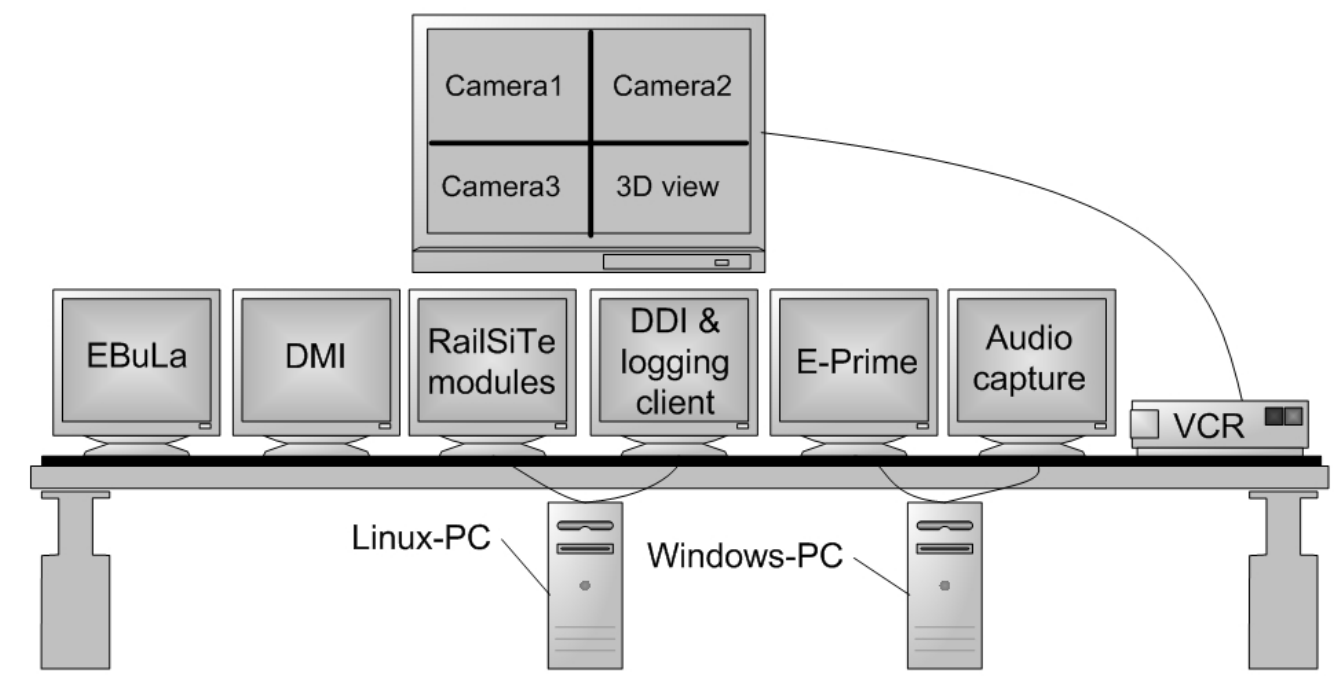

Figure 3: Schematic view of the supervisor desk

Principally, data sets are available as on-line version and also logged for later offline analysis. Furthermore, three independent audio channels between the driver and the supervisor were implemented: radio telephone communication (the supervisor acting as the signaller), general announcements by the supervisor to the subject and a continuously activated microphone in the driver's cabin.

Originally, the RailSiTe was used to test hardware devices for train control systems. So, it already supports a very detailed logging feature for all types of events. All log messages have a precise timestamp with microsecond granularity that can be later on used to synchronise the heterogeneous data types, i.e. audio and video files. The time stamp of a GPS time server is used for synchronisation. For the off-line processing and analysis of the data as well as for video coding, a special tool named AErogator that has been developed at the DLR Institute of Transportation Systems is used (Köster and Noyer, 2008).

For future studies concerning stress, the possibility to conduct a dual task was implemented. The driver has a special screen where different secondary tasks can be presented while he is driving. In summary, the supervisor desk allows for a complete observation of the driver and makes complex human factors studies possible for different purposes and scenarios.

\subsection{Driver's cab (Mockup)}

A closed test environment with reproducible conditions is vital to acquire comparable results. Thus, a closed driver's cabin had to be built to isolate the driver from the laboratory and increase the level of realism. 
The driver's desk was formerly positioned freestanding in the laboratory room. The acoustic and visual separations of the subject were the most important requirements for human factors studies. In order to give the driver the feeling of working in a closed room, the driver's cabin was constructed. Not only does the cabin offer a visual barrier to the possibly distracting laboratory surrounding and particularly the supervisor desk, but also the sound system will unfold a more realistic performance.

The cabin is an in-house manufacture work out of wooden and metal components. The interior was designed to match the original German train class ET 423. Since the cabin of this train type has large side windows, it was decided to additionally increase the level of realism by attaching displays to the sides. The front view is realised using a top-mounted video projector and a special widescreen-lens. Figure 3 shows the view of the driver through the front window. Finally, cameras and audio systems were installed in the mock-up in order to enable a proper communication and surveillance to the supervisor. The resulting cabin provides visual as well as acoustic isolation and produces a believable and constant test environment.

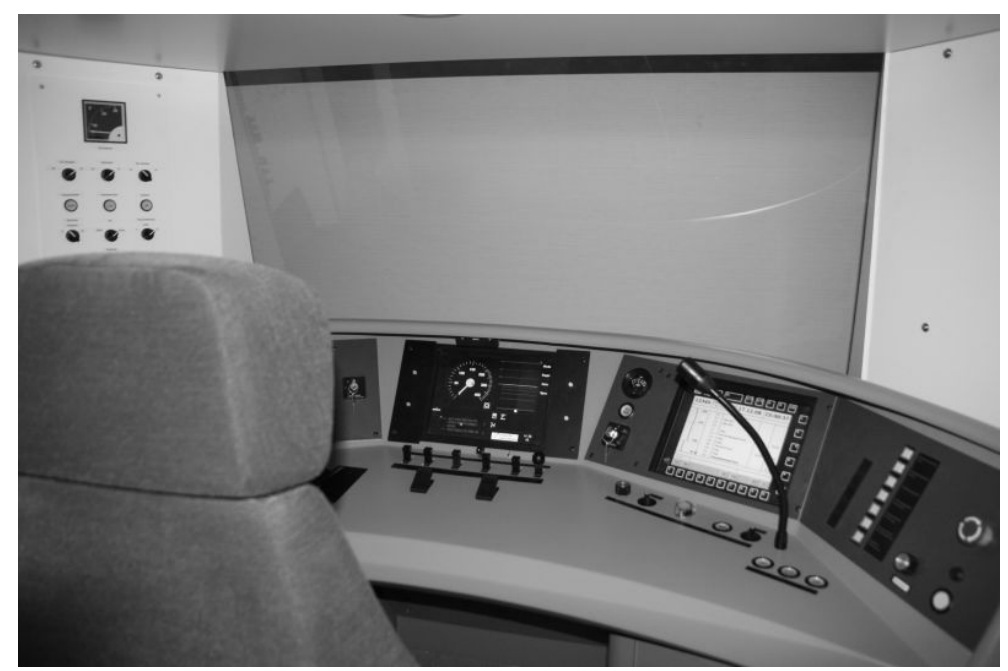

Figure 4: Interior view of the driver's cab

\subsection{Train control system and human-machine-interface}

In order to provide a realistic and consistent human-machine-interface, some of the driver's desk components had to be updated. Furthermore, in this step, the German intermittent train control system PZB was implemented in the laboratory. It was tried to simulate all interfaces and interactions to the driver subject in the way the equipment is installed and configured in practice.

In detail, the modifications involved the installation of a radio communication device. The original telephone handset enables the driver subject to initiate a train radio phone call to the local supervision centre. Here, these calls are connected to the supervisor.

A dead man's device was natively incorporated in the installation of the driver's desk. For the first studies, the device was configured to meet the frequency and dependencies of the German SIFA (Sicherheitsfahrschaltung) system. However, other options, e.g. the must to press the foot button continuously, can be set up easily.

In line with the proper simulation of the train control system PZB, the central drivermachine-interface was updated. The tool 2indicate is an in-house development of the German Aerospace Center to facilitate easy design of human-machine-interfaces. This software suite was used to model a multi-functional driver-machine-interface that is very common for modern train sets and locomotives. So, all indicator lamps of the SIFA and the so called PZB 90 can be integrated in the interface that is also showing a speedindicator and traction and braking force display. Fortunately, touch screen implementation of the DMI and the flexible tool 2indicate facilitate the adaptation for 
different interfaces.

Finally, the time table display (EBuLa) - on the right hand side in the driver's desk was updated to have the screen show the time table data available in the RailSiTe database. In a following step, the train-specific diagnosis display - on the left hand side in the driver's desk - will be implemented.

\subsection{Physiologic and eye-movement-measurements, visual surveillance}

For a comprehensive video surveillance, three cameras were installed into the driver cab. The requirements emerged to be very diverse. One camera targets the facial view. To capture eye blinks with a minimum duration (Häkkänen et al. 1999) a camera must have a sufficient frame rate. According to the Nyquist-Shannon sampling theorem (Shannon, 1998) a rate of 25 frames per second should be enough to cover these events. In contrast to the first camera, the second one has to cover a wide area of nearly two meters size from a short distance to get a view of the entire driver desk. Therefore, a wide angle of 110 degrees comes to use. The third camera gives a general view of the driver cab and accordingly the driver.

With the three cameras, the synchronisation of the videos is a complex issue. A video converter box avoids this problem by splitting a screen to quarters. The three videos together with a view of the 3D visualisation are captured as a single video in a split-screen.

For the measurement of psychological data, the HealthLab instruments by Koralewski are used that were already available in the institute. By this, the RailSiTe is able to record electrical skin resistance, heart rate, blood pressure etc. The time synchronization and Bluetooth connection between the master computer of the psychological measurement system and the RailSiTe were implemented.

To complement the surveillance tools, the latest addition to the driver cab is a system for eye movement measurements. With the implemented system Dikablis ${ }^{\mathrm{TM}} \mathrm{a}$ head and eye movement monitoring is possible. The system is suitable for longer experiments because of its very light head fixed unit. The eye-tracker allows for the identification of fixation areas and therefore is very useful for attention or perception studies.

\section{Capabilities of the new simulation environment}

The implementation of a fully functional train simulator establishes the possibility to conduct several experimental studies in the rail human factors domain. Nowadays, train drivers have to deal with an increase in complexity and information load in their workstation (e.g. by the addition of ETCS) combined with a high level of automated procedures that can lead to out-of-the-loop performance. Therefore, problems in the area of attention control, awareness and perception can arise that lead to an enhanced rate of mistakes or lapses. User-centred-design strives for the reduction of poor humanmachine-interaction.

Such situations can particularly occur when passing from one train control system to another, when entering a section with another signalling system or when traversing state borders and new rules apply. The high degree of automation also accounts to underload, fatigue and similar phenomena. When do these error favouring conditions appear and how can they be optimally countered? For the optimisation of railway safety, it is also to be studied how the human reliability can be positively influenced.

Modern railways are also interested in low energy consumption and an optimum of train connections and passenger changes in major stations. The disposition work is often centralized, together with the signaller's workplaces. How can the information on an energetically optimal driving profile and train connections be well communicated to the driver? Also, the human-machine-interface of potential assistant systems must be evaluated before introduction into the driver's cabin in practice.

Studies regarding the eye movements of train drivers in the mostly natural environment of a train simulator can help to clarify the limits of human perception 
processes and the problematic tasks or interfaces. In detail, fixation length or position can be used to reason the focus of attention, difficulties in perception or understanding of information, or memory processes.

For the redesign of human-machine-interfaces, possible research questions could be: What are the used information sources by the train driver for different tasks and points in time? Is there specific information in the display that can be blanked out without any performance decline? Which of the different HMI allows for rapid and correct understanding and action? The actual experimental session will be conducted with different interfaces where different levels of performance of the train driver can be recorded and analysed.

Other possible studies could include usability and HMI design, overload and stress, underload and fatigue as well as human reliability. The simulator is able to deal with all the different demands and therefore to be of important help in the investigation of rail human factors.

\section{Conclusion and outlook}

The article showed the modification process of the laboratory and the tools that the new simulation environment uses. The update enables the simulation of human factors for the train driver in a very realistic way.

Thereby, the origin of the laboratory in railway operation simulation was underlined: railway characteristics like train dynamics, interlocking and route setting are suitably simulated. In addition, the environment now offers and bunch of techniques for human factors subject studies. Some of them were highlighted in the last section.

The next research steps concern usability of existing interfaces and studies analysing the human-machine-interaction. Workload and human reliability assessment are in the focus of intermediate term analysis.

In the future, the full integration of the existing signaller's workplace is intended. Then, also staff communication issues can be addressed in the RailSiTe simulation environment.

\section{Acknowledgements}

This evolution would not have been possible without the continuous support by our research team. The authors are grateful to Frederike Feldmann, Matthias Grimm, Axel Rumke, Martin Busse, Andreas Gebauer and Thomas Knörr.

\section{References}

Häkkänen, H., Summala, H., Partinen, M., Tiihonen, M., Silvo, J., 1999. Blink duration as an indicator of driver sleepiness in professional bus drivers. Sleep (American Academy of Sleep Medecine, Rochester), 22, (6), 798 -802

Köster, F. and Noyer, U., 2008, Leistungsfähiges Datenmanagement als Rückgrat einer menschzentrierten Entwicklung von Automation/Assistenz. (High performing data management as the backbone of human-centred development of automation and assistance) 24. VDI/VW-Gemeinschaftstagung - Integrierte Sicherheit und Fahrerassistenzsysteme, 219-226

Jaschke, K.P., Hartwig, K., Meyer zu Hörste, M., Lemmer, K., 2007, A facility for testing ERTMS/ETCS conformity and human factors, In: J. Wilson et al. (ed.) People and Rail Systems: Human Factors at the Heart of the Railway (Ashgate publishing, London), 167-173

Knollmann, V., Obrenovic, M., Jäger, B., Lemmer, K., 2005, Optimized Migration Process in Rail Traffic Using Simulation Support. 1st International Seminar on Railway Operations Modelling and Analysis, Delft

Nash, A., Huerlimann, D., Schütte, J., Krauss, V.P., 2004, RailML - A standard data interface for railroad applications. In: Computers in Railways IX (WIT Press), 233-240 Shannon, C.E. (1998), Communication in the presence of noise, Proc. Institute of Radio Engineers, 37, (1), 10-21, 1949. Reprint as classic paper in Proc. IEEE, 86, (2) 